\title{
Study on Large-Scale Work Class ROV Based on Dynamic Analysis
}

\author{
Liu Xing ${ }^{2, a}$, Zhang $\mathrm{Ii}^{1}$, Wang Xinyuan ${ }^{1}$, Huang Suhe ${ }^{1}$, Sun Jian $^{1}$ \\ ${ }^{1}$ System Engineering Research Institute of CSSC, Beijing, China \\ ${ }^{2}$ Science and Technology on Underwater Acoustic Antagonizing Laboratory, Beijing, China \\ aliustar@gmail.com
}

Keywords: ROV, overall design, dynamic analysis

\begin{abstract}
ROV(Remotely Operated Vehicles) is widely used in ocean rescue and salvage, offshore oil mining, underwater engineering construction, military and national defense construction, etc, which has a broad application prospect. The paper design the structure and system frame type based on the successful experience of underwater robot. Then the propeller layout of underwater robot is study based on dynamic analysis.
\end{abstract}

\section{Introduction}

The ROV (Remotely Operated Vehicles) is a kind of device which can move in the water, with acoustic and optical sense system, by remote control, use the mechanical arm or other tools to finish underwater operations. It can be use in many fields such as oil, observation, construction and so on. The ROV obtains the operation control commands and power supply through the umbilical cable from surface console and uploads the working state and information data at the same time. The ROV can support complex underwater detection equipment and underwater operation machinery electricity. The transfer and exchange of large amount of information and data are quick and convenient.

The operation and control of ROV are operated by way of human-computer interaction from the surface. Because of the powerful computers, real-time control of underwater vehicle motion state and real-time observation of submersible and the target information of television images, the ability and the level of overall underwater observation and operation are high.

The ROV can be defined observation class and work class. The observation class ROV is used to observe and inspect the specific goals under water infrastructure, fisheries, hull, as well as scientific research projects and so on with small size and weight.The work class ROV has a large demands in ocean oil and gas, and there is no industrialization production in China; And which is expensive, has large profit space and Powerful functions. The work class ROV can be equipped with forward-looking sonar, side scan sonar, sounder, equipment with bigger size and weight and high cost.

The ROV technology has wide application in military field, mainly including coastal ports, coastal nuclear power plant, hydropower station, underwater security, underwater reconnaissance, examine, rescue, the mine warfare, and so on, also used in search and recovery of underwater loss of military equipment and weapons, such as torpedo. The application of the ROV system will accelerate the completion of tasks and system development process.

The paper design the structure and system frame type based on the successful experience of underwater robot with independent intellectual property rights. The ROV has a frame type structure and multiple propeller cooperate which completes the movement in all directions and the underwater operation is achieved by mechanical arm.

\section{The design of ROV platform frame structure}

Framework of each subsystem is the installed base of ROV which is used to withstand voltage electronic cabin, buoyancy material, hydraulic power unit, mechanical arm, and propeller etc.

At the same time, it is also the ROV main bearing structure for cloth, recycling and securing. 
The ROV platform framework scheme is an important factor to determine the ROV performance and the merits of the scheme will directly affect the overall performance of the ROV.

1) framework form

Frame type form is adopted with the main advantage of the convenient overall layout design and easy using and equipment changing.

Frame can protect and support the role of the important parts with running resistance increased. In the case of sufficient power, which can be remedied.

2) the system arrangement

Upper: mainly installed materials, ultra short baseline, mooring floating.

At the top of the center on both sides of the front-end and back-end layout four vertical thrusters which guarantee the complex current level of stability . Mooring position is located in the middle. Stem department installs cameras, searchlights.

Central: arrange the hydraulic system, electronic control system and horizontal thrusters to ensure the the forward thrust and resistance to movement speed.

4 propellers is decorated in the midship section which mainly used to ensure the ROV underwater movement and stability. In addition, in the stem department $2 \mathrm{~d}$ image sonar are installed.

Bottom: stem install removable mechanical arm and hydraulic power units, the communication control with pressure cabin are installed at the back

For the use of in underwater reef, anti- collision rubber strip can be used.

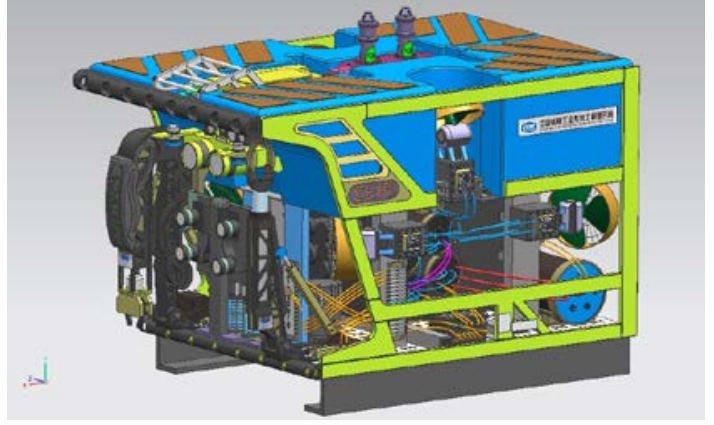

Figure 1 ROV system arrangement

\section{The ROV dynamics analysis}

The ROV should be implemented with lateral, dive and rotary four degrees of freedom of movement and the movement of the ROV are realized by the propeller. four propulsion thrusters in the horizontal and three propulsion thruster in the vertical are selected This scheme guarantee the mobility of ROV in horizontal and vertical submerged floating at the same time with the fewer thrusters.

Computational fluid dynamics method is used on the ROV hydrodynamic analysis. The domain is obtained by using the unstructured grid fills and the total number of grid is about 2.3 million, as shown in the figure below:
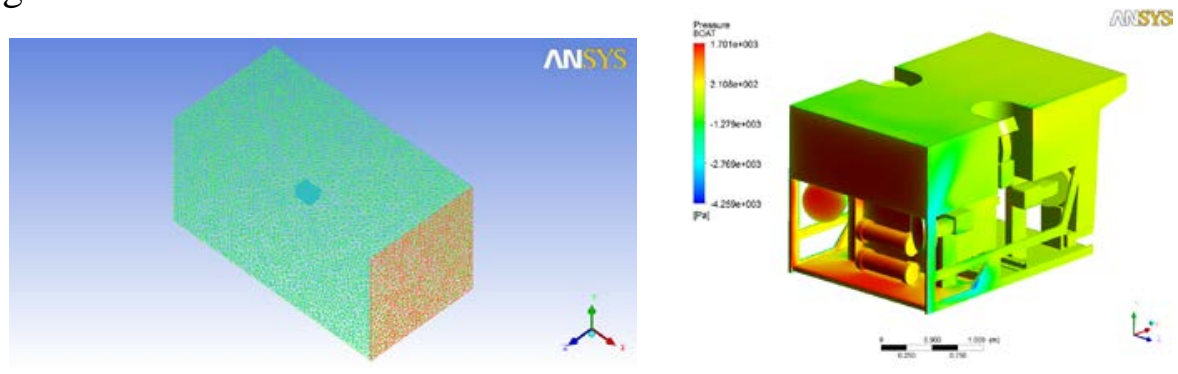

Figure 2 grid computing domain

Due to the asymmetry of the model, the movement are calculated twice, specific settings is as follows: 
- turbulence equation: $R N G k-\varepsilon$ equation;

- ingredients: water, density: $998.2 \mathrm{~kg} / \mathrm{m3}$, viscous: 0.001003;

down);

Entrance speed: $3.5 \mathrm{Kn}$ (forward and backward), $2.8 \mathrm{Kn}$ (left and right) and 2.2 Kn (up and

- boundary conditions: speed entry (water, air speed entrance), export pressure;

- equation discrete method: the pressure correction method use SIMPLEC method, with parameters of discrete and second-order accuracy of windward format. an adaptive grid technology is adopted.

The convergent curve as shown in figure 3:
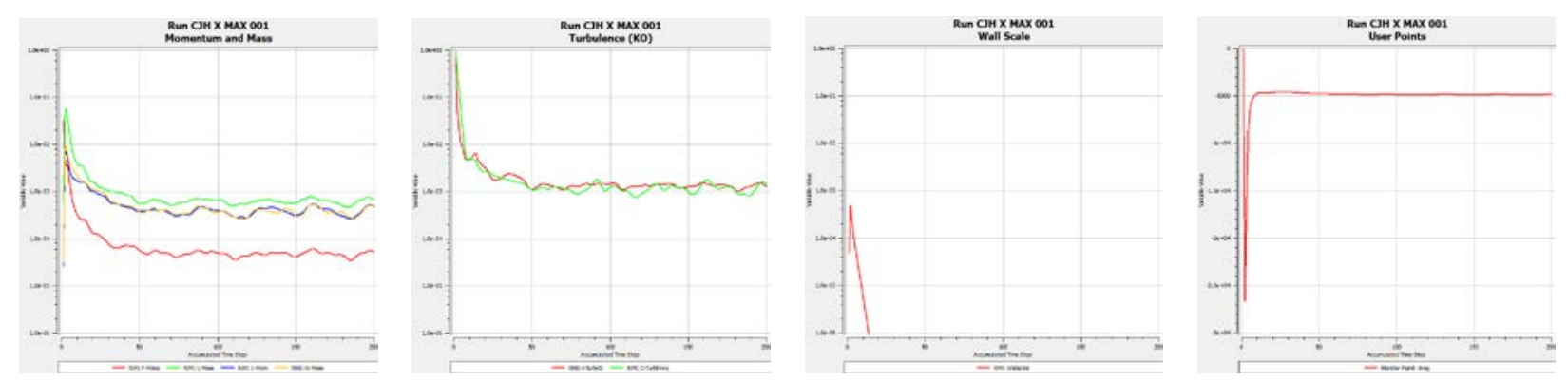

Figure 3 convergence curve of calculation process

(a) the convergent curve of calculation equation (b) the convergent curve of turbulence equation

(c) convergence curve of wall function (d) monitoring parameter curves

The ROV forward and backward movement speed is given as $3.5 \mathrm{Kn}$, up and down movement speed is given as $2.2 \mathrm{Kn}$, left and right movement speed is given as2.8 $\mathrm{Kn}$. The calculation results are as follows:
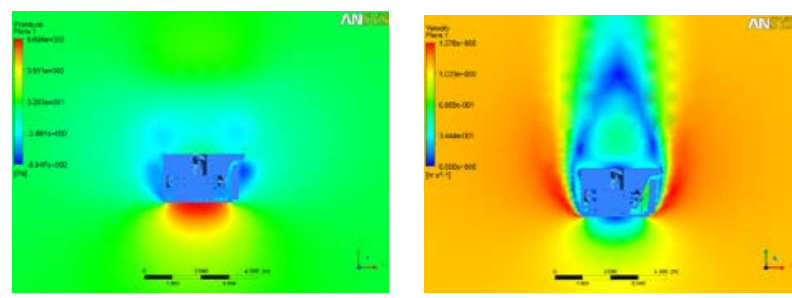

Figure 4 local stress nephogram and longitudinal symmetry plane (left) and local velocity contours (right).
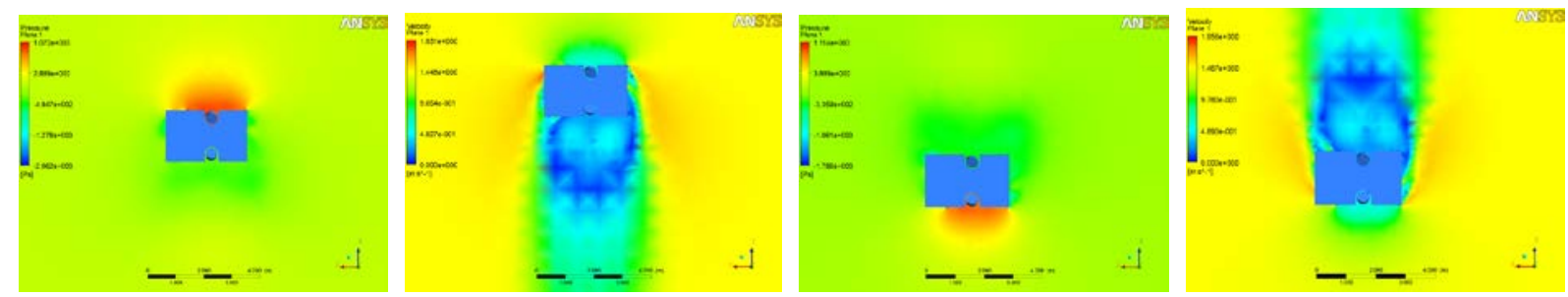

Figure 5 the partial pressure of water cloud picture (left) and local velocity contours (right).

The propulsion power need results by static conditions in the process of the ROV (use formula for $\mathrm{P}=\mathrm{F}^{*} \mathrm{~V}$ ) according to the CFD calculation is shown in table 2 .

Table 2 the hydrodynamic analysis of ROV

\begin{tabular}{|c|c|c|}
\hline Velocity /Kn & Resistance /N & Power /KW \\
\hline forward 3.5 & 4822.05 & 8.438588 \\
\hline backward3.5 & 4487.1 & 7.852425 \\
\hline Up 2.2 & 3581.83 & 3.940013 \\
\hline Down 2.2 & 3038.96 & 3.342856 \\
\hline left 2.8 & 4892.35 & 6.84929 \\
\hline right 2.8 & 4826.24 & 6.756736 \\
\hline
\end{tabular}

The result can be found by analysis table 3 that the power is large because of its high speed in 
forward backward movement and left right movement. Considering the efficiency of the propeller is made up of the overall efficiency (0.97), the relative rotation efficiency fuselage (0.93), car leaf efficiency (0.673). so the overall efficiency of about 0.559 . The layout direction for propeller is not parallel to the direction of motion, which provides the practical thrust is only $1 / \sqrt{2}$ of total thrust. So the efficiency of the propeller on the ROV is about 0.395.For the ROV equipped with seven propeller, 4 propellers can be used to provide the forward backward movement and left right movement. 4 propellers can be used to provide the up and down movement. According to the above analysis, the actual power is shown in table 4.

Table 4 the ROV propulsion power

\begin{tabular}{|c|c|c|}
\hline $\begin{array}{c}\text { Power of } \\
\text { forward and } \\
\text { backward (KW) }\end{array}$ & $\begin{array}{c}\text { Power of left } \\
\text { and right (KW) }\end{array}$ & $\begin{array}{c}\text { Power of up and } \\
\text { down (KW) }\end{array}$ \\
\hline 14.92 & 14.92 & 7.46 \\
\hline
\end{tabular}

From the table above, the selection of $8 \mathrm{kw}$ power propulsion motor is relatively reasonable and meet the requirements of ROV movement.

\section{Conclusion}

There are more than 270 species of ROV in the world and more than 400 companies which provide all kinds of ROV machine, components and service. The huge ROV industry has formed. The ROV are developing with a sound momentum in our country, but still keeps some pace with advanced level at home and abroad in the platform, detection, navigation and positioning technology. The domestic ROV is less.

With the process of development and utilization of Marine resources and the of mature of key technologies, it can be expected that the ROV will gain breakthrough in the field of operation ability, reliability, ability to adapt and more widely applied. ROV will play a more important role in the development and utilization of Marine resources, submarine detection, underwater engineering, underwater salvage, military and national defense construction. The research and development of ROV with independent intellectual property rights and the localization benefit the innovation ability in China and the level of manufacturing which adapt to the needs of coming peak demand and cultivate our underwater equipment technical strength.

\section{References}

[1] Bo Wang, Lei Wan, Yu-ru Xu, Zai-bai Qin. Modeling and simulation of a mini AUV in spatial motion[J]. Journal of Marine Science and Application . 2009 (1)

[2] McFarlane,.R. Underwater technology 2000 ROVs and AUVs: tools for exploring,exploiting and defending the ocean frontier. Underwater Technology,2000. UT00. Proceedings of the 2000 International SymPosium on. 2000: 465-471P

[3] AntonelliqT1Fossen,D Yoerger. Underwater Robotics in Handbook on Robotics [M]. Springer-Verlag, 2008

[4] Song, Feijun, An, P. Edgar, Folleco, Andres. Modeling and simulation of autonomous underwater vehicles: Design and implementation. IEEE Journal of Oceanic Engineering . 2003

[5] Evans J,Nahon M. Dynamic modeling and performance evaluation of an autonomous underwater vehicle. Ocean Engineering . 2004

[6] Xu Haijun, Pan Cunyun, Xie Haibin, Zhang Daibing. Dynamics modeling and simulation of a bionic swim bladder system in underwater robotics. Journal of Bionic Engineering . 2008

[7] Farhood Azarsina, Christopher D. Williams. Manoeuvring simulations of the MUN Explorer AUV based on the empirical hydrodynamics of axi-symmetric bare hulls. Applied Ocean Research. 2010 